\title{
QUALITY IMPROVEMENT Geriatrician-led care model in frail cardiology patients reduces re-admissions
}

\author{
Authors: James WM Henderson, ${ }^{A}$ Mark Sweeney, ${ }^{B}$ Melanie Dani ${ }^{C}$ and Shuli Levy ${ }^{D}$
}

\section{Background}

As the population admitted under cardiology is likely to become frailer, a geriatrician-led model of post-procedural care similar to that used in orthopaedic surgery may be beneficial.

\section{Methods}

In 2016, a new geriatrician-led ward was created in Hammersmith Hospital where frail cardiology patients could be transferred post-treatment. Using diagnostic coding, patients over the age of 65 years between 01 April and the 31 August for both 2016 and 2019 were identified, and data collected retrospectively from electronic patient records. An anonymised staff survey was completed following the introduction of the new service.

Results

Patients discharged from the geriatrician-led ward had fewer re-admissions than both cardiology-led wards in 2019 (chisquared 5.46; $p=0.02$ ), and overall re-admissions in 2016 (chisquared 4.34; $p=0.037$ ). The majority of surveyed respondents felt that this level of geriatrician input was useful.

\section{Conclusion}

Geriatrician-led post-procedural care in cardiology reduced 30-day re-admissions in an elderly cohort.

KEYWORDS: cardiology, geriatrics, care model, re-admissions

DOI: 10.7861/fhj.2021-0033

\section{Introduction}

Managing older people in acute hospitals poses specific challenges requiring multidisciplinary and holistic care. Older patients have higher rates of complications during their hospital stay and often have preventable hospital re-admissions. ${ }^{1,2}$ The importance of a multidisciplinary approach in orthopaedic surgery is well established, with a geriatric liaison service or shared care

Authors: Ajunior doctor, Imperial College Healthcare NHS Trust, London, UK; ${ }^{B}$ Wellcome Trust / NIHR clinical research fellow, Imperial College Healthcare NHS Trust, London, UK and Imperial College London, London, UK; ${ }^{C}$ consultant geriatrician, Imperial College Healthcare NHS Trust, London, UK and Imperial College London, London, UK; ${ }^{D}$ consultant geriatrician, Imperial College Healthcare NHS Trust, London, UK model recommended in the National Institute for Health and Care Excellence (NICE) guidelines for hip fractures in the older patient. ${ }^{3}$ Improvements in minimally invasive cardiology procedures mean that they are suitable for an increasingly frail population. ${ }^{4}$ We present a novel geriatrician-led model for cardiology patients at a large tertiary centre (Hammersmith Hospital).

In September 2016, a new ward was created to provide geriatrician-led post-procedural care for cardiology patients. This ward is led by geriatricians with a multidisciplinary team, tailored to meet the needs of the frailer patient. Once patients have received definitive cardiac treatment under the cardiology team, those who would benefit from geriatrician-led care can have their suitability recorded in the notes during the cardiology ward round. Patients are identified by the cardiology consultant as having complex discharge requirements, requiring further physical or occupational therapy input, or as being more frail and comorbid. These identified patients can then be highlighted at daily bed-flow meetings within the department, facilitating transfer. Patients still requiring definitive cardiac intervention or procedures, or requiring telemetry, are kept on the cardiology ward.

\section{Methodology}

Using procedural coding, all patients over the age of 65 years undergoing emergency angiography (with or without coronary intervention) or emergency pacing at Hammersmith Hospital between the 01 April and the 31 August for both 2016 and 2019 were identified. Baseline characteristics, length of stay and readmission within 30 days were then extracted retrospectively from patient records. For re-admission analysis, planned re-admissions for another procedure were not included. A 'cardiology' readmission was classed as one where the primary issue causing re-admission required either cardiology review, or a re-admission to the acute cardiology assessment unit. Length of stay was calculated from index procedure rather than from admission date to reduce the confounding effect of other improvements to the efficiency of the emergency treatment pathway. The staff survey data were performed in May 2017 following the implementation of the service, and was a voluntary, anonymised survey open to any team-member primarily working within the cardiology team. Statistical analysis was performed using R 4.0.1 (R Foundation for Statistical Computing, Vienna, Austria). Age and length of stay are presented as median (interquartile range (IQR)) as they are not normally distributed. Differences between re-admission rates and extended length of stay rates were tested for significance using a chi-squared test, and difference in median length of stay 
Table 1. Demographic and clinical characteristics of patients

\begin{tabular}{|c|c|c|c|c|}
\hline \multirow[t]{2}{*}{ Characteristic } & \multirow[t]{2}{*}{2016} & \multicolumn{3}{|l|}{2019} \\
\hline & & Cardiology-led discharge & Geriatrician-led discharge & 2019 total \\
\hline Age, years, median (IQR) & $80(77-84)$ & $80(77-83.5)$ & $84(80-88)$ & \\
\hline Male, $n(\%)$ & $147(60.0)$ & $101(66.0)$ & $31(36.9)$ & 132 \\
\hline Female, $\mathrm{n}(\%)$ & $98(40.0)$ & $52(34.0)$ & $53(63.1)$ & 105 \\
\hline PCI, n (\%) & $80(32.7)$ & $71(46.4)$ & $24(28.6)$ & 95 \\
\hline Pacemaker insertion, $\mathrm{n}(\%)$ & $74(30.2)$ & $33(21.6)$ & $36(42.9)$ & 69 \\
\hline Angiography without intervention, n (\%) & $89(36.3)$ & $47(30.7)$ & $24(28.6)$ & 71 \\
\hline ICD insertion, $n(\%)$ & $2(0.8)$ & $2(1.3)$ & $0(0.0)$ & 2 \\
\hline $\begin{array}{l}\text { Death while inpatient or within } 30 \text { days } \\
\text { of discharge, } n(\%)\end{array}$ & $9(3.7)$ & $12(7.5)$ & $1(1.2)$ & 13 \\
\hline Total, $\mathrm{n}(\%)$ & $245(100.0)$ & $153(64.6)$ & $84(35.4)$ & 237 \\
\hline
\end{tabular}

was tested using a two-tailed Mann-Whitney $U$ test. A p-value of $<0.05$ was considered statistically significant.

\section{Results}

In 2019, there were a total of 237 discharges of patients over 65 years; $84(35.4 \%)$ patients were discharged from the geriatricianled ward following an emergency procedure, compared with 153 $(64.6 \%)$ discharged directly from the cardiology-led ward. In the same period in 2016, there were 245 discharges, prior to the introduction of the geriatrician-led pathway. Table 1 presents a comparison of the patients' demographic and admission characteristics between 2016 and 2019, with 2019 being divided into two subgroups by the ward they were discharged from. For analysis of re-admissions and length of stay, patients who were transferred to a different team or to their local hospital (23 in 2016 and six in 2019), underwent a second procedure or planned re-admission ( 45 and 25), were treated privately (two and three) or died during their admission (nine and 13) were excluded from analysis, leaving 166 and 190 in total.

\section{Mortality}

In 2016, nine patients (3.7\%) died as an inpatient or within 30 days of admission, in comparison with $13(5.4 \%$ ) in 2019 , a non-significant difference (chi-squared $0.54 ; p=0.46$ ). Of those patients discharged from a geriatrician-led ward in 2019, one (1.2\%) patient died as an inpatient or within 30 days of admission, and of those discharged from a cardiology-led ward 12 (7.5\%) died as an inpatient or within 30 days of admission. There was no significant difference in the mortality rate between the patients who were discharged from a geriatrician-led ward and either the cardiology-led wards (chi-squared 3.44; $p=0.06$ ), or all patients in 2016 (chi-squared 0.60; $p=0.44$ ).

\section{Re-admissions}

Following the introduction of this model of care, the rate of re-admission from the geriatrician-led ward was significantly less than those discharged directly from a cardiology-led ward in 2019 (chi-squared 6.16; $p=0.01$ ), and overall re-admissions prior to the implementation of this pathway in 2016 (chi-squared 4.16; $p=0.041)$. Of the 82 included patients discharged from the geriatrician-led ward in 2019 , only two $(2.4 \%)$ patients were re-admitted within 30 days, compared with 15/108 (13.9\%) from the cardiology-led wards and $18 / 166$ overall $(10.8 \%)$ in 2016 . This is despite the geriatrician-led ward actively selecting the most complex and multimorbid patients.

Both re-admissions from the geriatrician-led ward in 2019 were for cardiological reasons, while seven re-admissions following direct discharge from the cardiology wards were due to general medical issues (with the remaining eight being for cardiological reasons). In 2016, four re-admissions were for general medical reasons, 13 were cardiological re-admissions and one was a readmission with pancreatitis which was managed by the surgical team.

\section{Length of stay}

Overall, despite the improvement in re-admissions rates, there was no increase in overall length of stay following the introduction of the pathway, with medians of 2 days (IQR 1-2) in 2016 vs 2 days (IQR 1-4) in 2019 ( $p=0.25$ ). In 2019, the median length of stay on the geriatrician-led ward was 2 days (IQR 1-4) in comparison with a median length of stay of 1 day (IQR 1-2) on the cardiologyled wards. As would be expected with a frailer and more complex population, the median length of stay on the geriatrician-led ward was significantly greater than both the cardiology-led ward in 2019 $(p<0.0001)$, and all patients in $2016(p<0.0001)$.

Six patients $(4 \%)$ in 2016, and four in $2019(2 \%)$ had an extended length of stay beyond 20 days which was not statistically different (chi-squared 0.290; $p=0.59$ ).

\section{Staff survey results}

There were 33 respondents in total to the anonymised staff survey: 15 nurses ( $45 \%$ ), eight cardiology consultants (24\%), five junior doctors (18\%), three occupational or physical therapists (10\%), and one healthcare assistant (3\%). Twenty-five (75.8\%) respondents agreed or strongly agreed with the statement: 'MoE [medicine of the elderly] input on A7 [cardiology ward] is beneficial 
to overall patient care and flow.' Twenty-four (72.7\%) respondents agreed or strongly agreed with the statement: 'Geriatrician-led input on C8 [geriatrician-led ward] is beneficial to frail/older cardiology patients.'

\section{Next steps}

We present a collaborative service in the care of older patients with cardiac issues. Our model of geriatrician-led care significantly reduced re-admissions within 30 days when compared with the cardiologist-led wards. There was no significant increase in mortality within 30 days of discharge or length of stay following the introduction of this service. The trend towards higher mortality on the cardiology-led ward was likely due to the fact that patients who were more unwell were kept on the cardiology-led ward due to the greater availability of monitoring, and to facilitate further intervention if required. The fact that there were no "general medical' re-admissions from the geriatrician-led ward implies there may be preventable re-admissions which can be avoided with the skills and expertise available in the multidisciplinary geriatric team. This is also reflected in some of the diagnoses prompting re-admission following discharge from the cardiology ward, which included 'constipation', 'reduced mobility' and 'fall' as the primary diagnoses underlying re-admission.

Although geriatric liaison consultation on complex patients was routine practice before the introduction of this new service, the switch to geriatrician-led care provides a new paradigm for their hospital journey. The model presented here was conceived and implemented based on strong informal positive feedback on our liaison service, combined with a belief within both teams that care could be improved for older patients by introducing a holistic model of care. This service change was received positively within the wider cardiology team, with the majority of surveyed staff agreeing or strongly agreeing that geriatrician-led care is beneficial to frail patients.

It may not be possible to implement this model in centres that lack the appropriate staffing or resources, who may wish instead to replicate some features within a liaison service. We would propose that the benefit in re-admissions presented here is derived mainly from the regular, structured multidisciplinary working that forms the basis of geriatric medicine practice. In our experience, frailty syndromes rarely improve following a single intervention or suggestion from a liaison service and are best managed with continuing care, surveillance and iteration from a named geriatrician. We would suggest, however, that regular multidisciplinary meetings incorporating some elements of the comprehensive geriatric assessment would be reproducible within a liaison model. Additionally, training of junior doctors and nurses to recognise and manage frailty-related issues such as delirium, polypharmacy and anticholinergic burden may help optimise patient care.

As longevity continues to improve across the globe, the population in Europe over the age of 70 is expected to increase by $41 \%$ by 2035 from the levels seen in $2015 .{ }^{5}$ Cardiovascular disease is primarily a disease of ageing, and with new interventions for older adults, including valve replacements and left ventricular assist devices, there will be increasing overlap between geriatric medicine and cardiology. ${ }^{4,6-8}$ Managing cardiac issues in this age group is fraught with challenges, including lack of relevant clinical guidelines, 'ageist' attitudes and a high prevalence of frailty and cognitive impairment. ${ }^{9-11}$ There have been increasing calls for 'geriatric cardiology' to be recognised as a cardiology subspecialisation in itself, however, it is also important to recognise the vast depth of experience and knowledge that already exists within the field of geriatric medicine. ${ }^{12}$

\section{Conclusion}

Geriatrician-led care of patients over the age of 65 years following an emergency cardiac procedure significantly reduced 30-day readmission rates. We propose that this collaborative model of care is the optimal model for managing frail older patients with cardiac issues. :

\section{References}

1 Brennan TA, Leape LL, Laird NM et al. Incidence of adverse events and negligence in hospitalized patients: results of the Harvard Medical Practice Study I. BMJ Qual Saf 2004;13:145-51.

2 Benbassat J, Taragin M. Hospital re-admissions as a measure of quality of health care: advantages and limitations. Arch Intern Med 2000;160:1074-81.

3 National Institute for Health and Care Excellence. Hip fracture: management: Clinical guideline [CG124]. NICE, 2017.

4 Eggebrecht $\mathrm{H}$, Mehta RH. Transcatheter aortic valve implantation (TAVI) in Germany 2008-2014: on its way to standard therapy for aortic valve stenosis in the elderly. EuroIntervention 2016;11:1029-33.

5 Nations United. World Population Prospects 2019. United Nations, 2019.

6 Forman DE, Rich MW, Alexander KP et al. Cardiac care for older adults: time for a new paradigm. J Am Coll Cardiol. 2011;57(18):1801.

7 Feldman T, Foster E, Glower DD et al. Percutaneous repair or surgery for mitral regurgitation. N Engl J Med 2011;364:1395-406.

8 Lampropulos JF, Kim N, Wang Y et al. Trends in left ventricular assist device use and outcomes among Medicare beneficiaries, 2004-2011. Open Hear 2014;1:e000109.

9 Arnett DK, Goodman RA, Halperin JL et al. AHA/ACC/HHS strategies to enhance application of clinical practice guidelines in patients with cardiovascular disease and comorbid conditions: from the American Heart Association, American College of Cardiology, and US Department of Health and Human Servi. J Am Coll Cardiol 2014:64:1851-6.

10 Bond M, Bowling A, McKee D et al. Does ageism affect the management of ischaemic heart disease? J Health Serv Res Policy 2003:8:40-7.

11 Dodson JA, Chaudhry SI. Geriatric conditions in heart failure. Curr Cardiovasc Risk Rep 2012;6:404-10.

12 Miller AP, Maurer M, Alexander KP. Geriatric cardiology: Two decades of progress and strategy for the future. J Am Coll Cardiol 2018;71:2970-3.

Address for correspondence: Dr James Henderson, Imperial Healthcare NHS Foundation Trust, Hammersmith Hospital, Du Cane Road, London W12 0HS, UK.

Email: james.henderson@doctors.org.uk 